EPJ Web of Conferences 73, 04009 (2014)

DOI: $10.1051 /$ epjconf/20147304009

(C) Owned by the authors, published by EDP Sciences, 2014

\title{
Meson baryon components in the states of the baryon decuplet
}

\author{
F. Aceti ${ }^{1, a}$, L.R. Dai ${ }^{2, b}$, L.S. Geng ${ }^{3, c}$, E. Oset ${ }^{1, d}$ and Y. Zhang ${ }^{2}$
}

${ }^{1}$ Departamento de Física Teórica and IFIC, Centro Mixto Universidad de Valencia-CSIC, Institutos de Investigación de Paterna, Apartado 22085, 46071 Valencia, Spain ${ }^{2}$ Department of Physics, Liaoning Normal University, Dalian 116029, China

${ }^{3}$ School of Physics and Nuclear Energy Engineering and International Research Center for Nuclei and Particles in the Cosmos, Beihang University, Beijing 100191, China

\begin{abstract}
We extend the Weinberg compositeness condition to partial waves of $L=1$ and resonant states to determine the weight of meson-baryon component in the $J^{P}=\frac{3}{2}^{+}$baryon decuplet. We obtain an appreciable weight of $\pi N$ in the $\Delta(1232)$ wave function, of the order of $60 \%$ and we also show that, as we go to higher energies in the members of the decuplet, the weights of meson-baryon component decrease and they already show a dominant part for a genuine component in the wave function. We interpret the meaning of the Weinberg sum-rule extended to complex energies.
\end{abstract}

\section{Introduction}

An interesting challenge in the study of the hadron spectrum, is understanding whether a resonance can be considered as a composite state of other hadrons or else a "genuine" state. An early attempt to answer this question was made by Weinberg in Ref. [1].

In a recent paper, the work was generalized to higher partial waves [2] and the results obtained were used to justify the commonly accepted idea that the $\rho$ meson is not a $\pi \pi$ composite state but a genuine one. The same method was also successfully used in Ref. [3] to evaluate the weight of composite $K \pi$ state in the $K^{*}$ wave function. However, no attempt was done to apply the method to baryonic resonances. We use it here to investigate the nature of the baryons of the $J^{P}=\frac{3}{2}^{+}$decuplet.

\footnotetext{
ae-mail: aceti@ific.uv.es

be-mail: dailr@lnnu.edu.cn

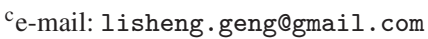

de-mail: oset@ific.uv.es
}

This is an Open Access article distributed under the terms of the Creative Commons Attribution License 4.0, which permits unrestricted use, distribution, and reproduction in any medium, provided the original work is properly cited. 


\section{The sum-rule for resonances and its interpretation}

As shown in Ref. [2], for a resonance or bound state dynamically generated by the interaction in coupled channels of two hadrons, the following relationship holds:

$$
-\sum_{i} \operatorname{Re}\left(g_{i}^{2}\left[\frac{d G_{i}}{d E}\right]_{E=\sqrt{s_{0}}}\right)=1-Z, \quad Z=\operatorname{Re} \int d^{3} p\left(\Psi_{\beta}(p)\right)^{2},
$$

where $\Psi_{\beta}(p)$ is the genuine component in the wave function of the state and $\sqrt{s_{0}}$ is the position of the resonance.

Eq. (1) is a generalization to complex energies of the sum-rule obtained in Ref. [2] for real energies. There it was interpreted as a consequence of the sum of probabilities of each channel to be unity. For complex values of the energies this interpretation is not possible and this is related to the fact that the modulus square of the wave function is substituted in Eq. (1) by the square of the wave function, chosen the stardand phase convention of Ref. [2]. Thus we should interpret $-g_{i}^{2} \frac{\partial G_{i}^{I I}}{\partial E}$ as the extrapolation of a probability into the complex plane, though it is not a probability. Yet, once we have interpreted it as the integrated strength of the wave function squared, we still can think of it as a magnitude providing the weight, or relevance of one given channel in the wave function of a state.

\section{Results}

\section{$3.1 \pi N$ scattering and the $\Delta(1232)$ resonance}

We use the sum-rule of Eq. (1) for the first time to investigate the nature of a baryonic resonance, the $\Delta(1232)$, in order to quantify the weight of $\pi N$ in this state.

\subsubsection{The model dependent test}

We first use a model based on chiral unitary theory. We fit the $\pi N$ data for the phase shifts using $t=\frac{1}{v^{-1}-G}$. The loop function $G$ is regularized by the cutoff $q_{\max }$ which is found from the best fit to the $\pi N$ data for the phaseshifts to be $452.6 \mathrm{MeV}$. The $G$ function is modified to contain the $|\vec{q}|^{2}$ factor (see Ref. [2] for more details). This choice is necessary for the generalization of the sum-rule for the couplings, found in Ref. [4] for the case of $s$-waves, to any partial wave.

In order to apply the sum-rule of Eq. (1) we need to extrapolate the amplitude to the complex plane. From the best fit, for the complex pole in the second Riemann sheet we find $\sqrt{s_{0}}=(1204.6+$ i44.37) $\mathrm{MeV}$ and for the coupling, which is obtained as the residue in the pole of the amplitude $t$, we get $\tilde{g}_{\Delta}=(8.53+i 1.85) \cdot 10^{-3} \mathrm{MeV}^{-1}$.

From these values we finally obtain $1-Z=0.62$, which indicates a sizeable weight of $\pi N$ in the resonance.

\subsubsection{The phenomenological test for the $\Delta(1232)$}

Now we perform a phenomenological test which makes use only of $\pi N$ scattering data.

We repeat the analysis of Refs. [2, 3] to test the sum-rule and we obtain $\sqrt{s_{0}}=(1208.00+$ $i 40.91) \mathrm{MeV}$ for the pole and $g_{\Delta}=(7.78+i 1.86) \cdot 10^{-3} \mathrm{MeV}^{-1}$ for the coupling of the $\Delta(1232)$, values which are very similar to those obtained with the procedure of the former subsection.

In this case we do not know the size of the cutoff $q_{\max }$ needed to regularize the derivative of $G$ in the second Riemann sheet, which is logarithmically divergent in the case of $p$-waves. Then, using natural values for the cutoff, as done in Refs. $[2,3]$, we try to establish the stability of the results in a certain 
Table 1. Values of $-g^{2} \frac{d G^{I I}}{d \sqrt{s}}$ and $1-Z$ for different cutoffs $q_{\max }$.

\begin{tabular}{lll}
\hline$q_{\max }[\mathrm{GeV}]$ & $-g^{2} \frac{d G^{I I}}{d \sqrt{s}}$ & $1-Z$ \\
\hline 0.4 & $0.47-i 0.38$ & 0.47 \\
0.5 & $0.57-i 0.29$ & 0.57 \\
0.6 & $0.65-i 0.22$ & 0.65 \\
\hline
\end{tabular}

Table 2. Poles, couplings, $-g^{2} \frac{d G^{I I}}{d \sqrt{s}}$ and $1-Z$ for the baryons of the decuplet $J^{P}=\frac{3}{2}^{+}$.

\begin{tabular}{lllllc}
\hline & Channel & $\sqrt{s_{0}}[\mathrm{MeV}]$ & $g\left[\mathrm{MeV}^{-1}\right.$ & $-g^{2} \frac{\partial G^{I I}}{\partial E}$ & $1-Z$ \\
\hline \multirow{2}{*}{$\Sigma(1385)$} & $\pi \Lambda$ & $1380.36+i 17.29$ & $(5.11+i 0.60) \cdot 10^{-3}$ & $(0.16-i 0.18)$ & 0.16 \\
& $\pi \Sigma$ & $1377.35+i 16.02$ & $(3.63+i 0.81) \cdot 10^{-3}$ & $(9.62-i 1.16) \cdot 10^{-2}$ & 0.10 \\
\hline$\Xi(1535)$ & $\pi \Xi$ & $1532.92+i 4.68$ & $(4.36+i 0.23) \cdot 10^{-3}$ & $0.11-0.09$ & 0.11 \\
\hline$\Omega^{-}$ & $\bar{K} \Xi$ & 1672.45 & $(1.56+i 0.37) \cdot 10^{-2}$ & 0.26 & 0.26 \\
\hline
\end{tabular}

range of $q_{\max }$. The values of $1-Z$ for three different values of $q_{\max }$ are shown in Table 1 . They are rather stable and consistent with the result obtained in the previous section.

\subsection{Application to other resonances}

Now we extend the study of the hadron-hadron content of resonances to the whole $J^{P}=\frac{3}{2}^{+}$baryons decuplet. We proceed as in the case of the $\Delta(1232)$, applying the phenomenological test of Sect. 3.1.2.

In order to evaluate the couplings of the resonances to the single channels, the branching ratios must be taken into account. Only the case of the $\Omega^{-}$is different, since this resonance is stable to strong decays. To apply the sum-rule we use a cutoff of the same order of magnitude of the one found in Sect. 3.1.1, $q_{\max } \simeq 450 \mathrm{MeV}$. The results obtained for the three resonances are shown in Table 2 .

\section{Conclusions and discussion}

We applied the generalized compositeness condition to the decuplet of the $\Delta(1232)$. We found the pole position for the $\Delta(1232)$ in very good agreement with the PDG [5] values.

We clarified the meaning of the extension of the Weinberg sum-rule for the case of resonances, finding that the concept of probability is changed to the squared of the wave function, within a certain phase convention, which, upon integration can be considered as a measure of the weight of a channel in the wave function.

As to the weight of the $\pi N$ component in the $\Delta(1232)$ wave function, we find relatively high values, of the order of $60 \%$. Yet, the result is less surprising when one recalls that from Drell Yan and deep inelastic scattering one induces a probability of about $34 \%$ for the $\pi N$ component in the nucleon. The large pion nucleon cloud in the $\Delta(1232)$ indicates that realistic calculations of its properties should take this cloud into account.

We extended the compositeness test to the other members of the decuplet and found a decreasing size of the meson-baryon components when we go to the $\Sigma(1385)$ and $\Xi(1535)$, indicating that these resonances are better represented by a genuine (in principle three quark) component. In the case of the $\Omega^{-}$we estimate the weight of the meson-baryon component to be small, of the order of $25 \%$. 
EPJ Web of Conferences

\section{References}

[1] S. Weinberg, Phys. Rev. 137, B672-B678 (1965)

[2] F. Aceti and E. Oset, Phys. Rev. D 86, 014012 (2012)

[3] C.W. Xiao, F. Aceti and M. Bayar, Eur. Phys. J. A 49, 22 (2013)

[4] D. Gamermann, J. Nieves, E. Oset and E. Ruiz Arriola, Phys. Rev. D 81, 014029 (2010)

[5] J. Beringer et al. [Particle Data Group Collaboration], Phys. Rev. D 86, 010001 (2012) 